\title{
Cluster Lifetime Analysis for Cooperative Beamforming in Wireless Sensor Networks
}

\author{
Gan Xiong ${ }^{1}$, Lu Hong ${ }^{1}$ and Yang Guangyou ${ }^{2}$ \\ ${ }^{1}$ School of Mechanical and Electronic Engineering, Wuhan University of \\ Technology, Wuhan, 430070, China \\ ${ }^{2}$ Institute of Agricultural Machinery, Hubei University of Technology, Wuhan, \\ 430068, China \\ ganxiong001@126.com
}

\begin{abstract}
Cooperative multiple input multiple outputs (MIMO) or its variation cooperative multiple input single outputs (MISO) is becoming a potential technique to minimize the energy consumption and prolong lifetime in wireless sensor networks. For cluster based wireless sensor networks, cluster lifetime for cooperative beamforming (CBF) based cooperative MISO scheme is studied in this paper. We first establish the energy model of a cluster with cooperative MISO scheme based on CBF. Based on the energy consumption model, the effects of cluster size and distance between cluster and sink node on the performance of cluster lifetime are discussed, an joint optimization to maximize cluster lifetime by finding the optimal modulation parameter and number of cooperative nodes is proposed. Numerical results show that CBF based cooperative MISO scheme can achieve longer cluster lifetime compare with STBC and the joint optimization can further improve the cluster lifetime.
\end{abstract}

Keywords: Cooperative MISO, Wireless Sensor Networks, Cluster Lifetime, Cooperative Beamforming, Joint Optimization

\section{Introduction}

Wireless sensor nodes are usually battery-powered, which are difficult or prohibitively expensive to replace or recharge. Thus, it is very important to improve the energy efficiency and prolong the lifetime in wireless sensor networks[1] .In order to improve energy efficiency ,wireless sensor networks are usually partitioned into clusters, cluster is consist of cluster head $(\mathrm{CH})$ and cluster members (CMs). CMs transmit data packets to the $\mathrm{CH}, \mathrm{CH}$ uses data aggregation algorithm to reduce the amount of data and transmits to sink node by single-hop or multi-hop[2].

Multiple input multiple output (MIMO) system requires less transmit power than single input single output (SISO) system under the same system performance requirements [3]. However, it may be difficult to equip each wireless sensor node with multiple antennas on its tiny operation platform. Cooperative MIMO scheme improves the signal strength at the receiver and reduce the transmit power by forming virtual antenna arrays. Cooperative MIMO scheme based on space time block code(STBC) scheme for wireless sensor networks is first proposed in [4].It is shown that cooperative MIMO can have more energy efficiency than SISO in long transmission distance. A more accurate energy consumption model considering the effect of increasing training overhead required in MIMO system is carried out in [5] and bit error rate is also statistical analyzed. Vertical bell labs layered space time(VBLAST) based cooperative MIMO communication architecture for wireless sensor networks is proposed in [6],VBLAST based cooperative MIMO scheme does not need sensor cooperation in transmitter side and provides significant energy savings over traditional SISO communication. Energy efficiency of 
CBF based cooperative MISO scheme is analyzed in [7], energy consumption is optimized by selecting the number of cooperative nodes (CNs) and cooperative MISO scheme base on CBF achieves more superiority than STBC in energy efficiency. In [8],the authors improve energy efficiency in a clustered wireless sensor network by combining cooperative MISO scheme base on STBC with data aggregation. Energy consumption of a cluster for STBC based cooperative MISO scheme is analyzed and cluster lifetime under different number of CNs is given in [9].Multi-hop cooperative MISO scheme is expanded in [10],hop distance and the number of CNs are jointly optimized to improve energy efficiency. Energy consumption per unit transmit distance for STBC based cooperative MISO scheme is optimized by selecting the number of CNs and the transmit energy consumption in [11].Energy efficient data gathering in wireless sensor networks by adopting cooperative MIMO is presented in [12].Even though these works have already showing the potential benefits of cooperative transmission in wireless sensor networks. However, in most of the above works are based on energy consumption analysis, rather than the view of lifetime. Meanwhile, the number of CNs or modulation parameter is set to be a fixed value, without considering joint optimization. The number of $\mathrm{CNs}$ is limit to 2 in $[4,5,12]$ and modulation size is a fixed value without considering rate optimization in [7-11].For these problems, cluster lifetime for CBF scheme is studied in this paper, the effects of cluster size and distance between cluster and sink node on the performance of cluster lifetime are discussed, an joint optimization to find the optimal modulation parameter and number of $\mathrm{CNs}$ to maximize cluster lifetime is proposed.

\section{System Model}

Scenarios considered in this paper are drilling platform monitoring, as each monitoring area could be considered as a cluster. To represent this environment, a simplified cluster model is used to do a concrete analysis. System model shown in figure 1, the network has a cluster and a sink node, $\mathrm{N}$ sensor nodes distributed in the cluster which the radius is $\mathrm{R}$, the distance between cluster and the sink node is $\mathrm{D}, \mathrm{D}>>\mathrm{R}$. Each node in the cluster is equipped with a single antenna and has EN $\mathbf{J}$ energy, perfectly synchronized transmission/reception between nodes and power control is enabled. For simplicity, sink node is also equipped with a single antenna and without energy limitation.

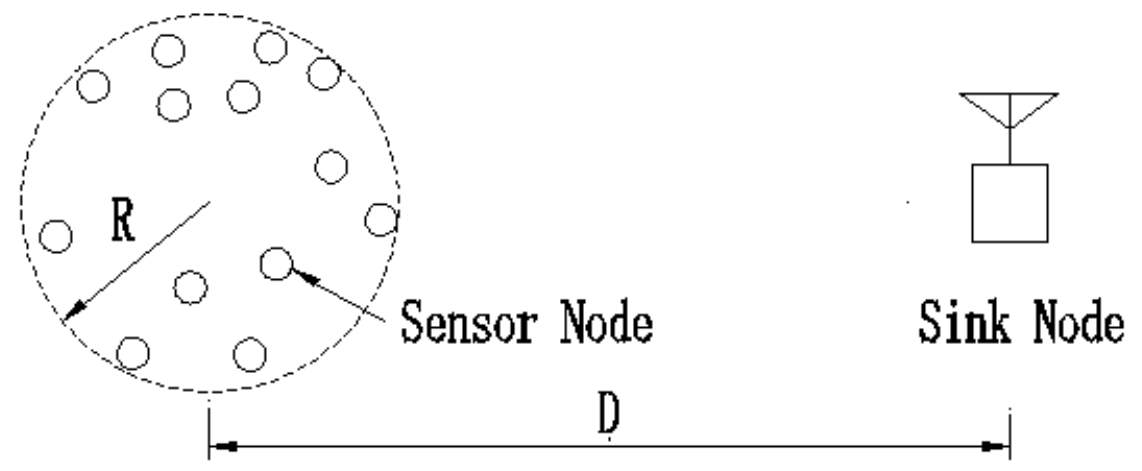

Figure 1. System Model

Each node in the cluster has $\mathrm{L}$ bit data that need to be transmitted to the sink node in each round. The cooperative MISO scheme based on CBF works in four phases as follows. 
Collecting phase: Sensor nodes in a cluster use different time slots to transmit their $\mathrm{L}$ bits data to $\mathrm{CH}, \mathrm{CH}$ can be any node in the cluster which has the most remaining energy.

Aggregating phase: $\mathrm{CH}$ in the cluster aggregates these data using linear aggregation with aggregation ratio $\gamma$.

Broadcasting phase: The $\mathrm{CH}$ broadcasts the compressed data to $\mathrm{M}$ CNs which have more remaining energies within the cluster. $(M=0$ means that broadcasting phase is not included and SISO scheme is used for the cooperative beamforming phase)

Cooperative beamforming phase: The $\mathrm{CH}$ and $\mathrm{CNs}$ in cluster encode the compressed data with the $\mathrm{CBF}$ scheme and cooperatively transmit them to the sink node.

\section{Energy Model}

A rayleigh fading channel with k-law path loss is assumed. The energy consumption of a cluster Etotal is the sum of the energy consumed in the four phases, which is given by:

$$
E_{\text {total }}=E_{c o l}+E_{a g g}+E_{b r o}+E_{c o o}
$$

where Ecol, Eagg, Ebro, Ecoo are the energy consumptions of the collecting, aggregating, broadcasting and cooperative beamforming phases, respectively.

Since $\mathrm{CH}$ and $\mathrm{CNs}$ are selected from all nodes in the cluster based on their remaining energies. To make steady data transmission, the transmission distance in collecting and broadcasting phases are assumed to be the worst case value 2R.Baseband signal processing blocks are intentionally omitted. The energy consumption in the collecting phase consist of the energy consumption of the power amplifier and the energy consumption of all other circuit blocks, can be represented as:

$$
E_{c o l}=\frac{(N-1) L}{R_{b}} P_{S I S O}+\frac{(N-1) L}{R_{b}}\left(P_{C T}+P_{C R}\right)
$$

The power consumption of the power amplifier PSISO can be represented as[4,13]:

$$
\begin{gathered}
P_{\text {SISO }} \approx \frac{\xi}{\eta} \bar{E}_{b_{-} S I S O} R_{b} \frac{(4 \pi)^{2}(2 R)^{k}}{G_{t} G_{r} \lambda^{2}} M_{l} N_{f} \\
\xi=3 \frac{\sqrt{2^{b}}-1}{\sqrt{2^{b}}+1}
\end{gathered}
$$

where $\xi$ is the Peak-to-Average Ratio (PAR) ; $\eta$ is the drain efficiency of the RF power amplifier; $\square$ Eb_SISO is the required average energy per bit at the receiver for a given BER requirement in SISO system; $R b=b B$ is the bit rate, $b$ is constellation size, $B$ is the system bandwidth; Gt is the transmitter antenna gain; $G r$ is the receiver antenna gain; $\lambda$ is the carrier wavelength; $\mathrm{Ml}$ is the link margin compensating the hardware process variations and other additive background noise or interference; $\mathrm{Nf}$ is the receiver noise figure.

The circuit power consumption at transmitter side PCT and the circuit power consumption at the receiver side PCR can be represented as[4,13]:

$$
\begin{gathered}
P_{C T}=P_{D A C}+P_{m i x}+P_{f i f t}+P_{s y n} \\
P_{C R}=P_{L N A}+P_{m i x}+P_{I F A}+P_{f i f r}+P_{A D C}+P_{s y n}
\end{gathered}
$$

Where PDAC, Pmix, Pfilt, Psyn, PLNA, PIFA, Pfilr, PADC are the power consumption value for the DAC, the mixer, the active filters at the transmitter side, the frequency synthesizer, the Low Noise Amplifier (LNA),the Intermediate Frequency Amplifier (IFA), the active filters at the receiver side and the ADC, respectively.

The energy consumption in the aggregating phase is given by:

$$
E_{\text {agg }}=L N E_{A G}
$$

where EAG is the energy consumption per bit for data aggregation. 
The energy consumption in the broadcasting phase is also contributed by the energy consumption of the power amplifier and the energy consumption of all other circuit blocks, can be represented as:

$$
E_{b r o}=q(M)\left(\frac{\gamma N L}{R_{b}} P_{S I S O}+\frac{\gamma N L}{R_{b}}\left(P_{C T}+M P_{C R}\right)\right)
$$

Where $q(M)=\left\{\begin{array}{ll}0 & , M=0 \\ 1 & , M \geq 1\end{array}\right.$.

The $\mathrm{CH}$ together with $\mathrm{CNs}$ in the cluster and the sink node to form a cooperative MISO system in cooperative beamforming phase. As the distance between the cluster and sink node $\mathrm{D}$ is usually much larger than the radius of the cluster $\mathrm{R}$, we assume that transmission distance in cooperative beamforming phase is $\mathrm{D}$. The energy consumption of the cluster in cooperative beamforming phase Ecoo is given by:

$$
E_{\text {coo }}=\frac{\gamma N L}{R_{b}} P_{M I S O}+\frac{\gamma N L}{R_{b}}(M+1) P_{C T}
$$

The power consumption of the power amplifier PMISO can be represented as:

$$
P_{\text {MISO }} \approx \frac{\xi}{\eta} \bar{E}_{b_{-} M I S O} R_{b} \frac{(4 \pi)^{2} D^{k}}{G_{t} G_{r} \lambda^{2}} M_{l} N_{f}
$$

Where $\square$ Eb_MISO is the required average energy per bit at the receiver for a given BER requirement in MISO system for CBF.

For BPSK modulation, the average BER $\square \mathrm{Pb}$ for CBF is given by[7]:

$$
\overline{P_{b}}=\left(\frac{1-S}{2}\right)^{M+1} \times \sum_{k=0}^{M} C_{M+k}^{k}\left(\frac{1+S}{2}\right)^{k}
$$

Where $S=\sqrt{\frac{\bar{\gamma}_{b}}{\bar{\gamma}_{b}+1}}, \bar{\gamma}_{b}$ is the average received SNR.

As BER requirements usually less than 10-3 and corresponds to a high SNR, $\sum_{k=0}^{M} C_{M+k}^{k}\left(\frac{1+S}{2}\right)^{k} \approx C_{2 M+1}^{M+1}$. The closed expression $\square$ Eb_MISO with BPSK modulation can be expressed as[11]:

$$
\bar{E}_{b_{-} M I S O}=N_{0} \bar{\gamma}_{b}=\frac{N_{0}}{\left(1-2\left(\frac{\bar{P}_{b}}{C_{2 M+1}^{M+1}}\right)^{\frac{1}{M+1}}\right)^{-2}-1}
$$

Especially, if $\mathrm{M}=0$, we get the required average energy per bit of BPSK modulation in SISO system $\square$ Eb_SISO, which can be expressed as:

$$
\bar{E}_{b_{-} S I S O}=\frac{N_{0}}{\left(1-2 \bar{P}_{b}\right)^{-2}-1}
$$

For MQAM modulation,the average BER $\square \mathrm{Pb}$ for CBF is given by[7]:

$$
\overline{P_{b}}=\frac{4}{b}\left(1-\frac{1}{2^{b / 2}}\right)\left(\frac{1-G}{2}\right)^{M+1} \times \sum_{k=0}^{M} C_{M+k}^{k}\left(\frac{1+G}{2}\right)^{k}
$$

Where $G=\sqrt{\frac{3 b \overline{\gamma_{b}} /\left(2^{b+1}-2\right)}{1+3 b \overline{\gamma_{b}} /\left(2^{b+1}-2\right)}}$.

When $b$ is odd, omit the term of $\left(1-2^{-\frac{b}{2}}\right)$ in (14).

Similarly, the closed expression $\square$ Eb_MISO with MQAM modulation can be expressed as: 


$$
\bar{E}_{b_{-} \text {MISO }}=N_{0} \bar{\gamma}_{b}=\frac{N_{0}\left(2^{b+1}-2\right)}{3 b\left(\left(1-2\left(\frac{b \bar{P}_{b}}{4\left(1-\frac{-b}{2}\right) C_{2 M+1}^{M+1}}\right)^{\frac{1}{M+1}}\right)^{-2}-1\right)}
$$

When $b$ is odd, omit the term of $\left(1-2^{-\frac{b}{2}}\right)$ in $(15)$.

For the same reason, the required average energy per bit of MQAM modulation in SISO system $\square$ Eb_SISO, which can be expressed as:

$$
\bar{E}_{b_{-} S I S O}=\frac{N_{0}\left(2^{b+1}-2\right)}{3 b\left(\left(1-\frac{b \bar{P}_{b}}{2\left(1-2^{-\frac{b}{2}}\right)}\right)^{-2}-1\right)}
$$

When $b$ is odd, omit the term of $\left(1-2^{-\frac{b}{2}}\right)$ in (16).

\section{Cluster Lifetime Analysis and Optimization}

In our model, selecting the $\mathrm{CH}$ and $\mathrm{CNs}$ in a cluster is based on their remaining energies. So, the energy consumption among all nodes in cluster can be regarded as balanceable and all nodes in a cluster have equal lifetime approximately[9]. So, cluster lifetime can be defined as:

$$
L_{\text {cluster }}=\frac{N E_{N}}{E_{\text {total }}}
$$

Proposition 1: For a given $\mathrm{b}$ and $\mathrm{M}$, Lcluster is a decreasing function of $\mathrm{R}$ and D,respectively.

Proof: $L_{\text {cluster }}=\frac{N E_{N}}{E_{\text {total }}}=\frac{N E_{N}}{E_{c o l}+E_{\text {agg }}+E_{\text {bro }}+E_{\text {coo }}}$

$\frac{\partial L_{\text {cluster }}}{\partial R}=\frac{-N E_{N}\left(\frac{\partial E_{c o l}}{\partial R}+\frac{\partial E_{b r o}}{\partial R}\right)}{E_{\text {total }}^{2}}, \frac{\partial E_{c o l}}{\partial R}=\frac{(N-1) L}{R_{b}} \frac{\partial P_{S I S O}}{\partial R}>0$,

$\frac{\partial E_{\text {bro }}}{\partial R}=q(M) \frac{\gamma N L}{R_{b}} \frac{\partial P_{\text {SISO }}}{\partial R} \geq 0$. As a result, $\frac{\partial L_{\text {cluster }}}{\partial R}<0$, Lcluster is a decreasing

function of $\mathrm{R}$ for a given $\mathrm{b}$ and $\mathrm{M}$.

$\frac{\partial L_{\text {cluster }}}{\partial D}=\frac{-N E_{N} \frac{\partial E_{c o o}}{\partial D}}{E_{\text {total }}^{2}}, \frac{\partial E_{\text {coo }}}{\partial D}=\frac{\gamma N L}{R_{b}} \frac{\partial P_{M I S O}}{\partial D}>0$. As a result, $\frac{\partial L_{\text {cluster }}}{\partial D}<0$, Lcluster is a decreasing function of $\mathrm{D}$ for a given $\mathrm{b}$ and $\mathrm{M}$.

Proposition 2: For a given $\mathrm{R}$ and $\mathrm{D}$, there exists an optimal combination (b, M) to maximize the cluster lifetime.

Proof: $L_{\text {cluster }}=\frac{N E_{N}}{E_{\text {total }}}=\frac{N E_{N}}{E_{C}+E_{P A}+E_{\text {agg }}}$

$E_{C}=\frac{L}{b B}\left((N-1+q(M) \gamma N+\gamma N M+\gamma N) P_{C T}+(N-1+q(M) \gamma N M+\gamma N(M+1)) P_{C R}\right)$

$E_{P A}=(N-1+q(M) \gamma N) L \frac{\xi}{\eta} \bar{E}_{b_{-} S I S O} \operatorname{Const}(2 R)^{k}+\gamma N L \frac{\xi}{\eta} \bar{E}_{b_{-} M I S O}$ ConstD $^{k}$

Const $=\frac{(4 \pi)^{2}}{G_{t} G_{r} \lambda^{2}} M_{l} N_{f}$

It can be found that, for a given R, D and $\mathrm{M}, \mathrm{EC}$ is a decreasing function of $\mathrm{b}$. From formula (4),(15) and (16), $\xi, \square \mathrm{Eb}$ SSISO and $\square \mathrm{Eb}$ MISO are a increasing function of $\mathrm{b}, \mathrm{EPA}$ is a increasing function of $\mathrm{b}$. As a result, there exists an optimal $\mathrm{b}$ for a given $\mathrm{R}, \mathrm{D}$ 
and M. Similarly, for a given R, D and b, EC is a increasing function of M, $\square$ Eb_MISO is a decreasing function of M, EPA is a decreasing function of $\mathrm{M}$. As a result, there exists an optimal $\mathrm{M}$ for a given $\mathrm{R}, \mathrm{D}$ and $\mathrm{b}$.

In general, there exists an optimal combination $(\mathrm{b}, \mathrm{M})$ to achieve cluster lifetime maximization for a given R, D. For a given system, the radius of cluster $\mathrm{R}$ and the distance between cluster and sink node $\mathrm{D}$ are also determined. Therefore, the optimization for the cluster lifetime can be characterized as:

$$
\begin{gathered}
(b, M)=\arg \max L_{\text {cluster }}(b, M) \\
\text { Subject to }: 1 \leq b \leq b_{\max } \\
0 \leq M \leq M_{\max }
\end{gathered}
$$

Where bmax and Mmax are the maximum value for constellation size and number of CNs, respectively.

The optimization belongs to the integer programming problem, since the search space is not too large, brute force search method is used to find the optimal values.

\section{Numerical Results}

Numerical results for cluster lifetime is shown in this section, the system parameters is shown in Table $1[4,9,13]$.

\section{Table 1. System Parameters}

\begin{tabular}{cccc}
\hline$\eta=0.35$ & $\mathrm{~B}=1 / \mathrm{Ts}=10 \mathrm{KHz}$ & $\mathrm{N} 0=-171 \mathrm{dBm} / \mathrm{Hz}$ & $\mathrm{GtGr}=5 \mathrm{dBi}$ \\
\hline$\lambda=0.12 \mathrm{~m}$ & $\mathrm{Ml}=40 \mathrm{~dB}$ & $\mathrm{Nf}=10 \mathrm{~dB}$ & $\square \mathrm{Pb}=10-3$ \\
\hline PDAC $=15.4 \mathrm{mw}$ & Pmix $=30.3 \mathrm{mw}$ & Pfifr $=2.5 \mathrm{mw}$ & Pfift $=2.5 \mathrm{mw}$ \\
\hline Psyn $=50 \mathrm{mw}$ & PLNA $=20 \mathrm{mw}$ & PIFA $=3 \mathrm{mw}$ & PADC $=6.70 \mathrm{mw}$ \\
\hline $\mathrm{N}=10$ & $\gamma=0.5$ & $\mathrm{EN}=40 \mathrm{~J}$ & $\mathrm{~L}=100 \mathrm{bits}$ \\
\hline $\mathrm{EAG}=5 \times 10-9 \mathrm{~J} / \mathrm{bit}$ & $\mathrm{k}=3$ & $\mathrm{bmax}=12$ & $\mathrm{Mmax}=3$ \\
\hline
\end{tabular}

Figure 2 shows the cluster lifetimes with different $\mathrm{R}$ when $\mathrm{D}=50 \mathrm{~m}, \mathrm{~b}=2$ for $\mathrm{M}=0,1,2,3$. The cluster has larger cluster size achieves smaller cluster lifetime. For instance, when $\mathrm{D}=50 \mathrm{~m}, \mathrm{~b}=2$ and $\mathrm{M}=1$, cluster lifetime decreases from $1.417 \times 104$ to $1.135 \times 104$ round as $\mathrm{R}$ increases from $2 \mathrm{~m}$ to $8 \mathrm{~m}$. The reason is that the increase of cluster size results in larger energy consumption in collecting and broadcasting phases and corresponds to smaller cluster lifetime.

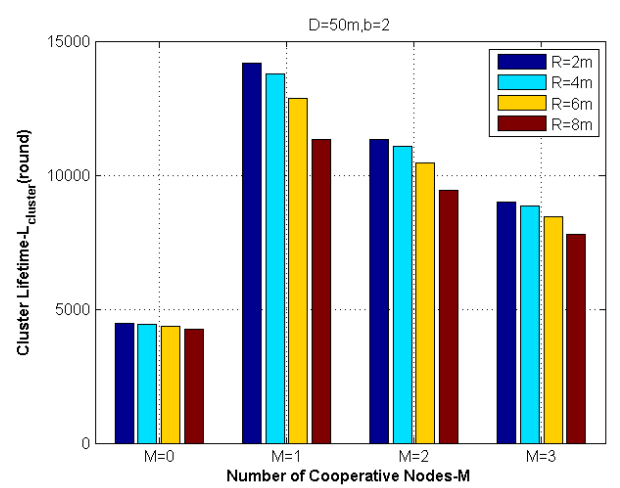

Figure 2. Cluster Lifetimes with Different $R$ when $D=50 \mathrm{~m}, b=2$ for $M=0,1,2$, 3

Figure 3 shows the cluster lifetimes with different $D$ when $R=5 m, b=2$ for $\mathrm{M}=0,1,2,3$. The cluster has larger cooperative transmission distance $\mathrm{D}$ achieves smaller cluster lifetime. For instance, when $\mathrm{R}=5 \mathrm{~m}, \mathrm{~b}=2$ and $\mathrm{M}=1$, cluster lifetime 
decreases from $1.534 \times 104$ to $0.944 \times 104$ round as $\mathrm{D}$ increases from $20 \mathrm{~m}$ to $80 \mathrm{~m}$. The reason is that the increase of cooperative transmission distance $\mathrm{D}$ results in larger energy consumption in cooperative beamforming phase and corresponds to smaller cluster lifetime.

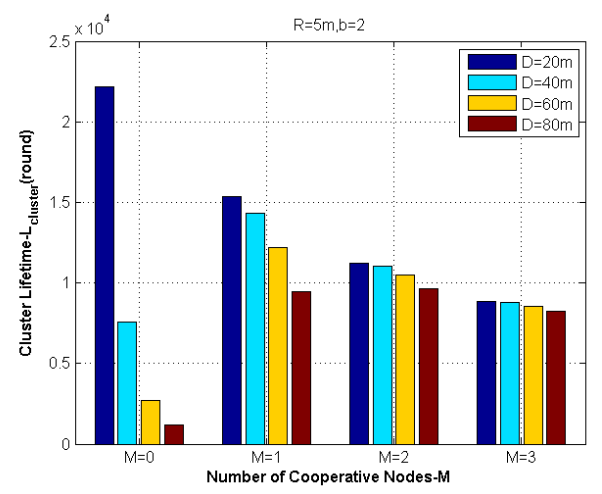

Figure 3. Cluster Lifetimes with Different $D$ when $R=5 m, b=2$ for $M=0,1,2,3$

Figure 4 shows cluster lifetimes with different $\mathrm{b}$ and $\mathrm{M}$ and when $\mathrm{R}=5 \mathrm{~m}, \mathrm{D}=50$ $\mathrm{m}$. For a given $\mathrm{b}$, there exists an optimal number of CNs. When $b=1$, the optimal number of $\mathrm{CNs}$ is 1 .For a given $\mathrm{M}$, there exists an optimal modulation parameter. When $\mathrm{M}=3$, the optimal modulation parameter is 4.In general, the optimal combination for modulation parameter and number of $\mathrm{CNs}$ is $(4,2)$ when $\mathrm{R}=5 \mathrm{~m}$, $\mathrm{D}=50 \mathrm{~m}$.

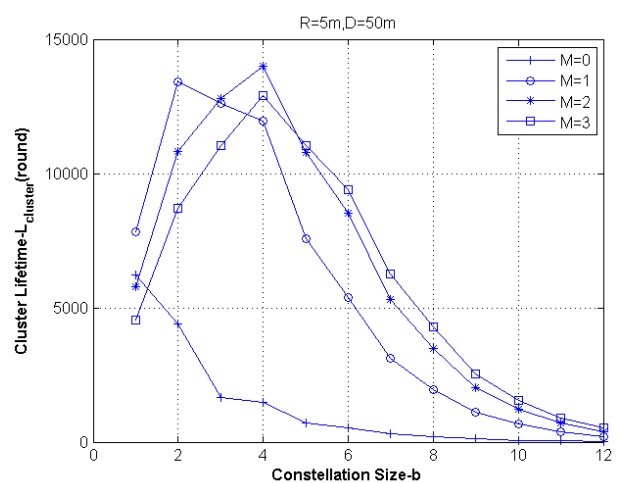

Figure 4. Cluster Lifetimes with Different $b$ and $M$ and when $R=5 \mathrm{~m}, D=50 \mathrm{~m}$

As shown in figure 5, joint optimization can extend the cluster lifetime over merely CNs optimization for both STBC and CBF schemes. As it can be seen, when $\mathrm{R}=5 \mathrm{~m}$ and $\mathrm{D}=100 \mathrm{~m}, 25 \%$ and $49 \%$ longer cluster lifetime are realized in STBC and CBF schemes, respectively.CBF scheme better than STBC under CNs optimization and joint optimization. However, $\mathrm{CBF}$ scheme can not achieve more superiority than STBC in CNs optimization when D less than $43 \mathrm{~m}$. The reason is that the optimal number of CNs is 0 and SISO is the optimal transmission scheme in that case. 


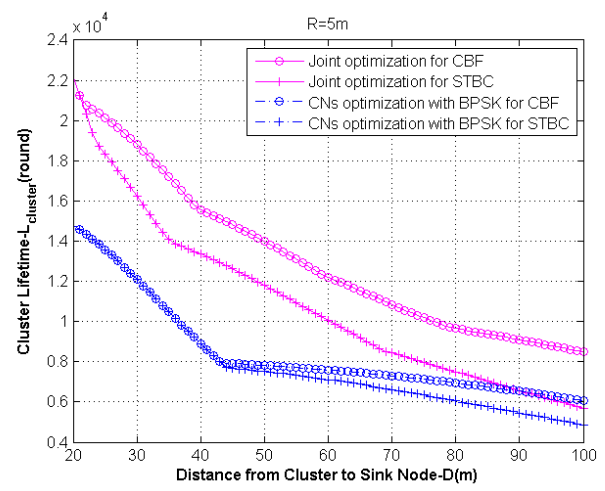

Figure 5. Cluster Lifetimes with/without Joint Optimization in STBC and CBF Schemes

\section{Conclusion}

Cluster lifetime for CBF based cooperative MISO scheme in wireless sensor network is analyzed in this paper. Theoretical analysis and numerical results show that, the larger of the cluster size and the distance between the cluster and the sink node, the smaller the cluster lifetime will be. Cooperative MISO scheme based on CBF can achieve longer cluster lifetime than STBC. Therefore, compared with merely CNs optimization, the joint optimization can further extend the cluster lifetime.

\section{Acknowledgements}

The work in this paper is supported by the National Natural Science Foundation of China (No.51174084 and No.51275372).

\section{References}

[1] T. Rault, A. Bouabdallah and Y. Challal, "Energy efficiency in wireless sensor networks: a top-down survey, Computer Networks", vol. 67, no. 8, (2014), pp.104-122.

[2] W. Heinzelman, A. Chandrakasan and H. Balarislman, "An application specific protocol architecture for wireless microsensor networks”, Wireless Communications, IEEE Transactions on, vol. 1, no. 4, (2002) , pp.660-670.

[3] D. N. Nguyen, M. Krunz, "Cooperative MIMO in wireless networks: recent developments and challenges", IEEE Network, vol. 27, no. 4, (2013), pp. 48-54.

[4] S. Cui, A. J. Goldsmith and A. Bahai, "Energy-efficiency of MIMO and cooperative MIMO techniques in sensor networks, Selected Areas in Communications, IEEE Journal on, vol. 22, no. 6, (2004), pp.1089-1098.

[5] S. K. Jayaweera, "Virtual MIMO-based Cooperative Communication for Energy-constrained Wireless Sensor Networks", Wireless Communications, IEEE Transactions on, vol. 5, no. 5, (2006), pp. 984-989.

[6] S. K. Jayaweera, "V-BLAST-based virtual MIMO for distributed wireless sensor networks", Communications, IEEE Transactions on, vol. 55, no. 10, (2007), pp. 1867-1872.

[7] J. C. Fan, Q. Y. Yin, W. J. Wang and A. Feng, "Analysis of energy efficiency for cooperative beamforming in wireless sensor networks", Journal on Communications, vol. 29, no. 11, (2008), pp. 145-151.

[8] Q. Gao, Y. Zuo, J. Zhang and X. H. Peng, "Improving energy efficiency in a wireless sensor network by combining cooperative MIMO with data aggregation", Vehicular Technology, IEEE Transactions on, vol. 59, no. 8, (2010), pp. 3956-3965.

[9] Z. Huang, K. Kobayashi, M. Katayama and T. Yamazato, "A study on cluster lifetime of single-hop wireless sensor networks with cooperative MISO scheme", IEICE transactions on communications, vol. 94, no. 10, (2011), pp. 2881-2885.

[10] J. Zhang, L. Fei, Q. Gao and X. H. Peng, "Energy efficient multihop cooperative MISO transmission with optimal hop distance in wireless ad hoc networks",Wireless Communications IEEE Transactions on, vol. 10, no. 10, (2011), pp. 3426-3435.

[11] B. Li, W. Wang, Q. Yin, R. Yang, Y. Li and C. Wang, "A new cooperative transmission metric in wireless sensor networks to minimize energy consumption per unit transmit distance, IEEE Communications Letters, vol. 16, no. 5, (2012), pp. 626- 629. 
[12] H. Xu, L. Huang, C. Qiao, W. Dai, and Y. Sun, "Joint Virtual MIMO and Data Gathering for Wireless Sensor Networks". Parallel and Distributed Systems, IEEE Transactions on, vol. 26, no. 4, (2015), pp. 1034-1048.

[13] S. Cui, A. J. Goldsmith and A. Bahai, "Energy-constrained modulation optimization, Wireless Communications, IEEE Transactions on, vol. 4, no. 5, (2005), pp. 2349-2360.

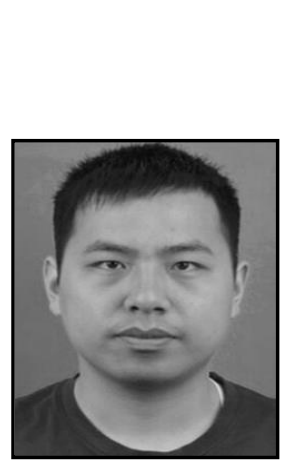

\begin{abstract}
Authors
Gan Xiong, he received the Master degree in Mechanical and Electrical Engineering from Hubei University of Technology in 2014. $\mathrm{He}$ is currently working toward the Ph.D. degree in the Wuhan University of Technology, majoring in Mechanical and Electrical Engineering. His research interests include wireless sensor networks, cooperative communication and intelligent networks.
\end{abstract}

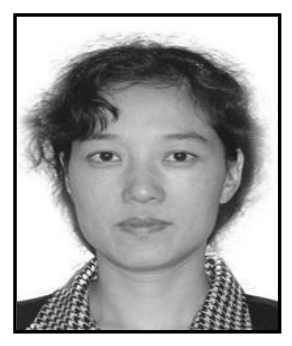

Lu Hong, she received the Master degree from Huazhong University of Science and Technology in 1990 and the Ph.D. Degree from the Wuhan University of Technology in 2000. She is currently as a professor of the school of Mechanical and Electrical Engineering, Wuhan University of Technology. Her research interests include network manufacturing and CAD/CAM.

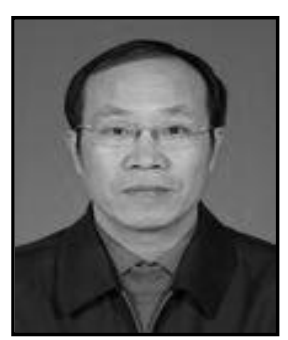

Yang Guangyou, he received the Master degree from Huazhong University of Science and Technology in 1990 and the Ph.D. Degree from the Wuhan University of Technology in 2006. He is currently as a professor of the Institute of Agricultural Machinery, Hubei University of Technology. His research interests include wireless sensor networks, embedded system and intelligent control. 
International Journal of Future Generation Communication and Networking Vol. 9, No.10, (2016) 